\title{
Light-Front Hamiltonian, Path Integral and BRST Formulations of the Chern-Simons-Higgs Theory in the Broken Symmetry Phase
}

\author{
Usha Kulshreshtha ${ }^{1 \#}$, Daya Shankar Kulshreshtha ${ }^{2}$, James P. Vary ${ }^{3}$ \\ ${ }^{1}$ Department of Physics, Kirori Mal College, University of Delhi, Delhi, India \\ ${ }^{2}$ Department of Physics and Astrophysics, University of Delhi, Delhi, India \\ ${ }^{3}$ Department of Physics and Astronomy, Iowa State University, Ames, USA \\ Email: "ushakulsh@gmail.com,dskulsh@gmail.com,jvary@iastate.edu
}

Received January 20, 2013; revised February 26, 2013; accepted March 9, 2013

Copyright (C) 2013 Usha Kulshreshtha et al. This is an open access article distributed under the Creative Commons Attribution License, which permits unrestricted use, distribution, and reproduction in any medium, provided the original work is properly cited.

\begin{abstract}
In the present work we study the Hamiltonian, path integral and BRST formulations of the Chern-Simons-Higgs theory in two-space one-time dimensions, in the so-called broken symmetry phase of the Higgs potential (where the phase $\varphi\left(x^{\mu}\right)$ of the complex matter field $\Phi\left(x^{\mu}\right)$ carries the charge degree of freedom of the complex matter field and is akin to the Goldstone boson) on the light-front (i.e., on the hyperplanes defined by the fixed light-cone time). The theory is seen to possess a set of first-class constraints and the local vector gauge symmetry. The theory being gauge-invariant is quantized under appropriate gauge-fixing conditions. The explicit Hamiltonian and path integral quantization is achieved under the above light-cone gauges. The Heisenberg equations of motion of the system are derived for the physical degrees of freedom of the system. Finally the BRST quantization of the system is achieved under appropriate BRST gauge-fixing, where the BRST symmetry is maintained even under the BRST light-cone gauge-fixing.
\end{abstract}

Keywords: Light-Front Quantization; Hamiltonian Quantization; Path Integral Quantization; BRST Quantization; Constrained Dynamics; Gauge Symmetry; Chern-Simons-Higgs Theory; Broken Symmetry Phase; Higgs Potential; Spontaneous Symmetry Breaking

\section{Introduction}

Gauge theories in two-space one-time dimensions involving Chern-Simons (CS) term coupled to matter fields describe excitations with fractional statistics [1-8]. Such studies form a broad field of study [1-15].

The Hamiltonian [16], path integral [17-19] and Becchi, Rouet, Stora and Tyutin (BRST) [20-22], formulations of the pure CS theory have been studied in Refs. $[7,8]$, in the instant-form (IF) quantization (IFQ) $[23,24]$ as well as in the light-front (LF) quantization (LFQ) $[23,24]$.

The CS theory in the presence of a Higgs potential has

*Talk presented by UK at the "International Conference on Light-Cone 2011: Applications of Light-Front Coordinates to Highly Relativistic Systems," held at the Southern Methodist University, Dallas Campus, Dallas, USA, May 22-27, 2011 (Published in Conf. Proceedings (Few Body Syst. 52, 457-461 (2012)).

${ }^{*}$ Corresponding author. been studied in Refs. [9-14], under appropriate gaugefixing conditions, in the so-called symmetry phase of the Higgs potential [9-11] as well as in the so-called broken (or frozen) symmetry phase $[12,13]$ of the Higgs potential [12-15], where the phase $\varphi\left(x^{\mu}\right)$ of the complex matter field $\Phi\left(x^{\mu}\right)$ carries the charge degree of freedom of the complex matter field and is, in fact, akin to the Goldstone boson [12-15].

The IF Hamiltonian, path integral and BRST formulations of this theory have been studied in Ref. [12] on the hyperplanes defined by the IF time: $x^{0}=t=$ constant $[23,24]$, in the broken (or frozen) symmetry phase of the Higgs potential [12-15] under appropriate gauge-fixing conditions.

In this work we study the LF Hamiltonian, path integral and BRST formulations of this CS-Higgs (CSH) theory on the hyperplanes defined by the light-cone (LC) time: 


$$
\left(\tau \equiv x^{+}=\frac{\left(x^{0}+x^{1}\right)}{\sqrt{2}}=\text { constant }\right)
$$

$[13,23,24]$, in the BSP of the Higgs potential.

This is in contrast to our earlier work [12] on the quantization of the same theory, where we have studied the IFQ of this theory in the BSP of the Higgs potential. In the present work, on the other hand, we study its LFQ.

We further wish to emphasize that the preliminary results of this work on the LFQ of this theory are presented in Ref. [13] and the complete details of this work are described here in the present work.

It may be important to mention here that the LFQ of any theory has several advantages over its IFQ for several well known reasons [18,19,23,24]. Also because the LF coordinates are not related to the conventional IF coordinates by a finite Lorentz transformation, the descriptions of the same physical result may be different in the IF and LF dynamics. In fact, the study of a theory using the IFQ as well as the LFQ determines the canonical structure and the constrained dynamics of the theory rather completely $[18,19,23,24]$. These are the main motivations for our present studies.

Also, different aspects of this theory have been studied by several authors in various contexts [1-8]. For further details about the motivations for a study of the different aspects of the $\mathrm{CSH}$ theories by various authors including a comparative description of different studies, we refer to the work of Refs. [1-8], as well as to our earlier work of Refs. [7-13].

In this work we present the complete details of the LFQ of this theory in the broken (or frozen) symmetry phase of the Higgs potential (cf. the work of Ref. [13] for our preliminary results). After a brief recap of the theory in the broken (or frozen) symmetry phase of the Higgs potential in the next section, its LF Hamiltonian and path integral formulations are presented in Section 3 and its LF BRST formulation is described in Section 4. The summary and discussion is finally given in Section 5 .

\section{Theory in Broken (or Frozen) Symmetry Phase: A Recap}

The Chern-Simons-Higgs theory in two-space one-time dimensions is defined by the action [1-15]:

$$
\begin{aligned}
& S_{1}=\int \mathcal{L}_{1}\left(\Phi, \Phi^{*}, A^{\mu}\right) \mathrm{d}^{3} x \\
& \mathcal{L}_{1}=\left[\frac{\kappa}{2} \epsilon^{\mu \nu \lambda} A_{\mu} \partial_{v} A_{\lambda}+\left(\tilde{D}_{\mu} \Phi^{*}\right)\left(D^{\mu} \Phi\right)-V\left(|\Phi|^{2}\right)\right] \\
& V\left(|\Phi|^{2}\right)=\alpha_{0}+\alpha_{2}|\Phi|^{2}+\alpha_{4}|\Phi|^{4} \\
& V\left(|\Phi|^{2}\right)=\lambda\left(|\Phi|^{2}-\Phi_{0}^{2}\right)^{2}, \Phi_{0} \neq 0
\end{aligned}
$$

$$
\begin{aligned}
& D_{\mu}=\left(\partial_{\mu}+i e A_{\mu}\right), \tilde{D}_{\mu}=\left(\partial_{\mu}-i e A_{\mu}\right), \\
& \kappa=\left(\frac{\theta}{2 \pi^{2}}\right) \\
& g^{\mu v}:=\operatorname{diag}(+1,-1,-1), \\
& \mu, \nu=0,1,2, \epsilon^{012}=\epsilon_{012}=+1
\end{aligned}
$$

Here $\theta$ is the Chern-Simons parameter. We keep the Higgs potential rather general, i.e., without making any specific choice for the parameters of the potential except that they are chosen such that the potential remains a double well potential with $\Phi_{0} \neq 0$. This action thus describes the theory in the so-called symmetry phase [9-11]. In the following we however, study this theory in the socalled broken (or frozen) symmetry phase (BSP) [12-15], of the complex matter field $\Phi\left[\equiv \Phi\left(x^{\mu}\right)\right]$. For this purpose, for the complex matter field $\Phi$ we take [12-15]:

$$
\Phi\left(x^{\mu}\right)=\Phi_{0}\left[\exp \left(i \varphi\left(x^{\mu}\right)\right)\right], \Phi_{0} \neq 0
$$

Here $\varphi\left[\equiv \varphi\left(x^{\mu}\right)\right]$ is the phase of the complex matter field $\Phi\left(x^{\mu}\right)$. The acion of the theory in the BSP $[3,4,7]$ then becomes:

$$
\begin{aligned}
S & =\int \mathcal{L} \mathrm{d}^{3} x, \mathcal{L} \\
& :=\left[\frac{\kappa}{2} \epsilon^{\mu v \lambda} A_{\mu} \partial_{v} A_{\lambda}+\frac{1}{2}\left(\partial_{\mu} \varphi+e A_{\mu}\right)\left(\partial^{\mu} \varphi+e A^{\mu}\right)\right]
\end{aligned}
$$

It is important to notice here that the vector gauge boson $A^{\mu}$ becmes massive in the BSP. This mass generation of the vector gauge boson takes place perhaps through a mechanism similar to the Higgs mechanism [9-15]. The phase $\varphi$ carries the charge degree of freedom of $\Phi$ and is, in fact, akin to the Goldstone boson and is to be treated as a dynamical field. Also the ground state in the BSP is not rotational invariant. Such studies of the theory in the broken-symmetry (superfluid) state could be relevant for the effective theories in the condensed matter, as the action of the theory describes the low-lying excitations in the BSP [12-15], as well as for an understanding of the issue of exotic statistics in gauge-invariant observables [1-8]. In the next section we study the LF Hamiltonian and path integral formulations of the above theory in the BSP.

\section{Light-Front Hamiltonian and Path Integral Formulations}

The LF action of the theory in the BSP of the Higgs potential reads:

$$
S=\int \mathcal{L} \mathrm{d} x^{+} \mathrm{d} x^{-} \mathrm{d} x_{2}
$$




$$
\begin{aligned}
\mathcal{L} & :=\left[\frac{\kappa}{2}\left(A^{+} \partial_{+} A_{2}-A^{-} \partial_{-} A_{2}+A^{-} \partial_{2} A^{+}-A^{+} \partial_{2} A^{-}-A_{2} \partial_{+} A^{+}+A_{2} \partial_{-} A^{-}\right)\right. \\
& \left.+\left(\partial_{+} \varphi\right) \partial_{-} \varphi+e\left(A^{-} \partial_{-} \varphi+A^{+} \partial_{+} \varphi\right)+e^{2} A^{+} A^{-}-\frac{1}{2}\left(e A_{2}+\partial_{2} \varphi\right)^{2}\right]
\end{aligned}
$$

Canonical momenta obtained from the above action are:

$$
\begin{aligned}
& \pi=\frac{\partial L}{\partial\left(\partial_{+} \varphi\right)}=\left(\partial_{-} \varphi+e A^{+}\right) \\
& \Pi^{+}=\frac{\partial \mathcal{L}}{\partial\left(\partial_{+} A^{-}\right)}=0, \Pi^{-}=\frac{\partial \mathcal{L}}{\partial\left(\partial_{+} A^{+}\right)}=-\frac{\kappa}{2} A_{2} \\
& E\left(\equiv \Pi^{2}\right)=\frac{\partial \mathcal{L}}{\partial\left(\partial_{+} A_{2}\right)}=\frac{\kappa}{2} A^{+}
\end{aligned}
$$

Here $\pi, \Pi^{+}, \Pi^{-}$and $E\left(=\Pi^{2}\right)$ are the momenta canonically conjugate respectively to $\varphi, A^{-}, A^{+}$and $A_{2}$. The above equations however, imply that the theory possesses four primary constraints:

$$
\begin{aligned}
& \chi_{1}=\Pi^{+} \approx 0, \chi_{2}=\left(\pi-\partial_{-} \varphi-e A^{+}\right) \approx 0 \\
& \chi_{3}=\left(\Pi^{-}+\frac{\kappa}{2} A_{2}\right) \approx 0, \chi_{4}=\left(E-\frac{\kappa}{2} A^{+}\right) \approx 0
\end{aligned}
$$

The symbol $\approx$ here denotes a weak equality (WE) in the sense of Dirac [16], and it implies that these above constraints hold as a strong equality only on the reduced hypersurface of the constraints and not in the rest of the phase space of the classical theory (and similarly one can consider it as a weak operator equality (WOE) for the corresponding quantum theory) $[16,18,19]$. The canonical Hamiltonian density corresponding to $\mathcal{L}$ is:

$$
\begin{aligned}
\mathcal{H}_{c} & :=\left[\pi \partial_{+} \varphi+\Pi^{+} \partial_{+} A^{-}+\Pi^{-} \partial_{+} A^{+}+E \partial_{+} A_{2}-L\right] \\
& =\left[\frac{\kappa}{2}\left[A^{-} \partial_{-} A_{2}-A^{-} \partial_{2} A^{+}+A^{+} \partial_{2} A^{-}-A_{2} \partial_{-} A^{-}\right]-e A^{-} \partial_{-} \varphi-e^{2} A^{+} A^{-}+\frac{1}{2}\left(e A_{2}+\partial_{2} \varphi\right)^{2}\right]
\end{aligned}
$$

After including the primary constraints $\chi_{1}, \chi_{2}, \chi_{3}$ and $\chi_{4}$ in the canonical Hamiltonian density $\mathcal{H}_{c}$ with the help of the Lagrange multiplier field $s, u, v$ and $w$ the total Hamiltonian density $\mathcal{H}_{T}$ could be written as:

$$
\begin{aligned}
\mathcal{H}_{T}= & {\left[\left(\Pi^{+}\right) s+\left(\pi-\partial_{-} \phi-e A^{+}\right) w+\left(\Pi^{-}+\frac{\kappa}{2} A_{2}\right) u+\left(E-\frac{\kappa}{2} A^{+}\right) v\right.} \\
& \left.+\frac{\kappa}{2}\left[A^{-} \partial_{-} A_{2}-A^{-} \partial_{2} A^{+}+A^{+} \partial_{2} A^{-}-A_{2} \partial_{-} A^{-}\right]-e A^{-} \partial_{-} \varphi-e^{2} A^{+} A^{-}+\frac{1}{2}\left(e A_{2}+\partial_{2} \varphi\right)^{2}\right]
\end{aligned}
$$

The Hamilton's equations of motion of the theory that preserve the constraints of the theory in the course of time could be obtained from the total Hamiltonian (and are omitted here for the sake of bravity): $H_{T}=\int \mathcal{H}_{T} \mathrm{~d}^{2} x$. Demanding that the primary constraint $\chi_{1}$ be preserved in the course of time, one obtains the secondary Gausslaw constraint of the theory as:

$$
\chi_{5}=\left(e \partial_{-} \varphi+e^{2} A^{+}\right) \approx 0
$$

The preservation of $\chi_{2}, \chi_{3}, \chi_{4}$ and $\chi_{5}$, for all times does not give rise to any further constraints. The theory is thus seen to possess only five constraints $\chi_{i}$ (with $i=1$, $2,3,4,5)$, where $\chi_{1}, \chi_{2}, \chi_{3}$ and $\chi_{4}$ are primary constraints and $\chi_{5}$ is a secondary constraint. Further, the matrix of the Poisson brackets among the constraints $\chi_{i}$, with $(i=1,2,3,4,5)$ is seen to be a singular matrix implying that the set of constraints $\chi_{i}$ is first-class and that the theory under consideration is gauge-invariant.
The physical degrees of freedom of the system are governed by the reduced Hamiltonian density of the theory (which is obtained by implementing the constraints of the theory strongly) [8-13]. Also, in the present case, $A^{+}$, and $\varphi$ play the role of gauge variables and the two pairs $\left(A^{-}, \Pi^{+}\right)$and $\left(A_{2}, E\right)$ are the pair of inessential eliminable variables and a pair describing the physical degrees of freedom of the system. Accordingly, we choose, in the present case, the first pair namely, $\left(A^{+}, \Pi^{-}\right)$as the pair describing the physical degrees of freedom and the other pair as the pair of inessential eliminable variables. So for writing the reduced Hamiltonian density of the theory, we choose $\varphi, \pi, \Pi^{*}, A^{-}$and $\Pi^{+}$as the independent variables and the remaining phase space variables as the dependent variables. The later ones are then expressed in terms of the independent variables as:

$$
\Pi^{+}=0, E=\frac{\kappa}{2} A^{+}, A_{2}=-\frac{2}{\kappa} \Pi^{-}, \pi=\left(\partial_{-} \varphi+e A^{+}\right)
$$


Finally the reduced Hamiltonian density of the theory describing the physical degrees of freedom of the system expressed in terms of the independent variables is then obtained as:

$$
\begin{aligned}
& \mathcal{H}_{R}= \\
& {\left[\Pi^{-} \partial_{-} A^{-}+\kappa A^{+} \partial_{2} A^{-}-\frac{\kappa}{2} e^{2} A^{+} A^{-}+\frac{1}{2}\left(\partial_{2} \varphi-\frac{2 e}{\kappa} \Pi^{-}\right)^{2}\right](11)}
\end{aligned}
$$

where $H_{R}=\int \mathcal{H}_{R} \mathrm{~d}^{2} x$ is the reduced Hamiltonian of the theory and it describes the physical degrees of freedom of the system. Here we reminded ourselves that as an alternative to the above, we could have equivalently expressed it in terms of the other pair namely, $\left(A_{2}, E\right)$ instead of the pair $\left(A^{-}, \Pi^{+}\right)$. The field equations derived from the Heisenberg equations of motion are then obtained as:

$$
\begin{aligned}
& -\partial_{+} \pi=i\left[\pi, H_{R}\right]=\left[-\partial_{2} \partial_{2} \varphi+\frac{2 e}{\kappa} \partial_{2} \Pi^{-}\right] \\
& \partial_{+} \varphi=-i\left[\varphi, H_{R}\right]=0 \\
& -\partial_{+} \Pi^{+}=i\left[\Pi^{+}, H_{R}\right]=\left[-\partial_{-} \Pi^{-}-\kappa \partial_{2} A^{+}-\frac{\kappa}{2} e^{2} A^{+}\right] \\
& \partial_{+} A^{-}=-i\left[A^{-}, H_{R}\right]=0 \\
& -\partial_{+} \Pi^{-}=i\left[\Pi^{-}, H_{R}\right]=\left[\kappa \partial_{2} A^{-}-\frac{\kappa}{2} e^{2} A^{-}\right] \\
& \partial_{+} A^{+}=-i\left[A^{+}, H_{R}\right]=\left[\partial_{-} A^{-}+\frac{4 e^{2}}{\kappa^{2}} \Pi^{-}-\frac{2 e}{\kappa} \partial_{2} \varphi\right]
\end{aligned}
$$

The vector gauge current of the theory

$$
\begin{aligned}
& J^{\mu} \equiv\left(J^{+}, J^{-}, J^{2}\right) \text { is: } \\
& J^{+}=\int j^{+} \mathrm{d} x^{-} \mathrm{d} x_{2} \\
& =\int \mathrm{d} x^{-} \mathrm{d} x_{2}\left[e \beta\left(\partial_{-} \varphi+e A^{+}\right)+\frac{\kappa}{2}\left(A_{2} \partial_{-} \beta-A^{+} \partial_{2} \beta\right)\right] \\
& J^{-}=\int j^{-} \mathrm{d} x^{-} \mathrm{d} x_{2} \\
& =\int \mathrm{d} x^{-} \mathrm{d} x_{2}\left[e \beta\left(\partial_{+} \varphi+e A^{-}\right)-\frac{\kappa}{2}\left(A_{2} \partial_{+} \beta-A^{-} \partial_{2} \beta\right)\right] \\
& J^{2}=\int j^{2} \mathrm{~d} x^{-} \mathrm{d} x_{2} \\
& =\int \mathrm{d} x^{-} \mathrm{d} x_{2}\left[-e \beta\left(\partial_{2} \varphi+e A_{2}\right)-\frac{\kappa}{2}\left(A^{-} \partial_{-} \beta+A^{+} \partial_{+} \beta\right)\right]
\end{aligned}
$$

The divergence of the vector gauge current density of the theory could now be easily seen to vanish satisfying the continuity equation: $\partial_{\mu} j^{\mu}=0$, implying that the theory possesses at the classical level, a local vector-gauge symmetry. The action of the theory is indeed seen to be invariant under the local vector gauge trans- formations:

$$
\begin{aligned}
& \delta \varphi=e \beta, \delta A^{-}=-\partial_{+} \beta, \delta A_{2}=-\partial_{2} \beta, \delta \Pi^{+}=0 \\
& \delta A^{+}=-\partial_{-} \beta, \delta E=\frac{-\kappa}{2} \partial_{-} \beta, \delta \Pi^{-}=\frac{\kappa}{2} \partial_{2} \beta, \delta \pi=0 \\
& \delta s=-\partial_{+} \partial_{+} \beta, \delta u=e \partial_{+} \beta, \delta w=-\partial_{+} \partial_{-} \beta \\
& \delta v=-\partial_{+} \partial_{2} \beta, \delta \Pi_{s}=\delta \Pi_{u}=\delta \Pi_{v}=\delta \Pi_{w}=0
\end{aligned}
$$

where $\beta \equiv \beta\left(x^{+}, x^{-}, x^{2}\right)$ is an arbitrary function of its arguments. In order to quantize the theory using Dirac's procedure we now convert the set of first-class constraints of the theory $\eta_{i}$ into a set of second-class constraints, by imposing, arbitrarily, some additional constraints on the system called gauge-fixing conditions or the gauge constraints. For this purpose, for the present theory, we could choose, for example, the following gauge-fixing condition: $\Psi=A^{-} \approx 0$. Here the gauge $A^{-} \approx 0$ represents the light-cone coulomb gauge and is a physically important gauge. Corresponding to this gauge choice, the theory has the following set of constraints under which the quantization of the theory could e.g. be studied:

$$
\begin{aligned}
& \xi_{1}=\eta_{1}=\chi_{1}=\Pi^{+} \approx 0 \\
& \xi_{2}=\eta_{2}=\chi_{2}=\left(\pi-\partial_{-} \varphi-e A^{+}\right) \approx 0 \\
& \xi_{3}=\eta_{3}=\chi_{3}=\left(\Pi^{-}+\frac{\kappa}{2} A_{2}\right) \approx 0 \\
& \xi_{4}=\eta_{4}=\chi_{4}=\left(E-\frac{\kappa}{2} A^{+}\right) \approx 0 \\
& \xi_{5}=\eta_{5}=\chi_{5}=\left(e \partial_{-} \varphi+e^{2} A^{+}\right) \approx 0 \\
& \xi_{6}=\Psi=A^{-} \approx 0
\end{aligned}
$$

The matrix $R_{\alpha \beta}$ of the Poisson brackets among the set of constraints $\xi_{i}$ with $(i=1,2,3,4,5,6)$ is seen to be nonsingular with the determinant given by

$$
\begin{aligned}
& {\left[\left\|\operatorname{det}\left(R_{\alpha \beta}\right)\right\|\right]^{\frac{1}{2}}} \\
& =\left[\left[e \kappa \delta^{2}\left(x^{-}-y^{-}\right)\right]\left[\partial_{-} \delta\left(x^{-}-y^{-}\right)\right]\left[\delta^{3}\left(x_{2}-y_{2}\right)\right]\right]
\end{aligned}
$$

The other details of the matrix $R_{\alpha \beta}$ are omitted here for the sake of bravity. Finally, following the standard Dirac quantization procedure, the nonvanishing equal light-cone-time commutators of the theory, under the gauge: $A^{-} \approx 0$ are obtained as:

$$
\begin{aligned}
& {\left[\varphi\left(x^{+}, x^{-}, x_{2}\right), \pi\left(x^{+}, x^{-}, x_{2}\right)\right]} \\
& =2 i \delta\left(x^{-}-y^{-}\right) \delta\left(x_{2}-y_{2}\right)
\end{aligned}
$$




$$
\begin{aligned}
& {\left[A^{+}\left(x^{+}, x^{-}, x_{2}\right), \Pi^{-}\left(x^{+}, x^{-}, x_{2}\right)\right]} \\
& =\frac{i}{2} \delta\left(x^{-}-y^{-}\right) \delta\left(x_{2}-y_{2}\right) \\
& {\left[A_{2}\left(x^{+}, x^{-}, x_{2}\right), E\left(x^{+}, x^{-}, x_{2}\right)\right]} \\
& =\frac{i}{2} \delta\left(x^{-}-y^{-}\right) \delta\left(x_{2}-y_{2}\right) \\
& {\left[\varphi\left(x^{+}, x^{-}, x_{2}\right), A_{2}\left(x^{+}, x^{-}, x_{2}\right)\right]} \\
& =\frac{i e}{2 \kappa} \epsilon\left(x^{-}-y^{-}\right) \delta\left(x_{2}-y_{2}\right)
\end{aligned}
$$

$$
\begin{aligned}
& {\left[A^{+}\left(x^{+}, x^{-}, x_{2}\right), A_{2}\left(x^{+}, x^{-}, x_{2}\right)\right]} \\
& =\frac{-i}{\kappa} \delta\left(x^{-}-y^{-}\right) \delta\left(x_{2}-y_{2}\right) \\
& {\left[E\left(x^{+}, x^{-}, x_{2}\right), \Pi^{-}\left(x^{+}, x^{-}, x_{2}\right)\right]} \\
& =\frac{i \kappa}{4} \delta\left(x^{-}-y^{-}\right) \delta\left(x_{2}-y_{2}\right)
\end{aligned}
$$

Also, for the later use, for considering the BRST formulation of the theory we convert the total Hamiltonian density into the first-order Lagrangian density $L_{I 0}$ :

$$
\begin{aligned}
\mathcal{L}_{I 0} & :=\left[\pi\left(\partial_{+} \varphi\right)+\Pi^{+}\left(\partial_{+} A^{-}\right)+\Pi^{-}\left(\partial_{+} A^{+}\right)+E\left(\partial_{+} A_{2}\right)+\Pi_{s}\left(\partial_{+} s\right)+\Pi_{u}\left(\partial_{+} u\right)+\Pi_{v}\left(\partial_{+} v\right)+\Pi_{w}\left(\partial_{+} w\right)-\mathcal{H}_{T}\right] \\
& =\left[\frac{\kappa}{2}\left[-A^{-} \partial_{-} A_{2}+A^{-} \partial_{2} A^{+}-A^{+} \partial_{2} A^{-}+A_{2} \partial_{-} A^{-}\right]-\frac{1}{2}\left(e A_{2}+\partial_{2} \varphi\right)^{2}+\left(\partial_{-} \varphi+e A^{+}\right) \partial_{+} \varphi-\frac{\kappa}{2} A_{2} \partial_{+} A^{+}+\frac{\kappa}{2} A^{+} \partial_{+} A_{2}\right]
\end{aligned}
$$

In the path integral formulation, the transition to quantum theory is made by writing the vacuum to vacuum transition amplitude for the theory called the generating functional $Z\left[J_{k}\right]$ of the theory [17-19] under the gauge-fixing under consideration, in the presence of the external sources $J_{k}$ as:

$$
Z\left[J_{k}\right]=\int[\mathrm{d} \mu] \exp \left[i \int \mathrm{d}^{3} x\left[J_{k} \Phi^{k}+\pi \partial_{+} \varphi+\Pi^{+} \partial_{+} A^{-}+\Pi^{-} \partial_{+} A^{+}+E \partial_{+} A_{2}+\Pi_{s} \partial_{+} s+\Pi_{u} \partial_{+} u+\Pi_{v} \partial_{+} v+\Pi_{w} \partial_{+} w-\mathcal{H}_{T}\right]\right]
$$

Here, the phase space variables of the theory are: $\Phi^{k} \equiv\left(\varphi, A^{-}, A^{+}, A_{2}, s, u, v, w\right)$ with the corresponding respective canonical conjugate momenta:

$$
\Pi_{k} \equiv\left(\pi, \Pi^{+}, \Pi^{-}, E, \Pi_{s}, \Pi_{u}, \Pi_{v}, \Pi_{w}\right) .
$$

The functional measure $[\mathrm{d} \mu]$ of the generating functional $Z\left[J_{k}\right]$ under the above gauge-fixing is obtained as:

$$
\begin{aligned}
{[\mathrm{d} \mu] } & =\left[e \kappa \delta^{2}\left(x^{-}-y^{-}\right)\right]\left[\partial_{-} \delta\left(x^{-}-y^{-}\right)\right]\left[\delta^{3}\left(x_{2}-y_{2}\right)\right][\mathrm{d} \varphi]\left[\mathrm{d} A^{+}\right]\left[\mathrm{d} A^{-}\right]\left[\mathrm{d} A_{2}\right] \\
& \times[\mathrm{d} s][\mathrm{d} u][\mathrm{d} v][\mathrm{d} w][\mathrm{d} z][\mathrm{d} \pi]\left[\mathrm{d} \Pi^{-}\right]\left[\mathrm{d} \Pi^{+}\right][\mathrm{d} E]\left[\mathrm{d} \Pi_{s}\right]\left[\mathrm{d} \Pi_{u}\right]\left[\mathrm{d} \Pi_{v}\right]\left[\mathrm{d} \Pi_{w}\right] \\
& \times \delta\left[\Pi^{+} \approx 0\right] \delta\left[\left(\Pi^{-}+\frac{\kappa}{2} A_{2}\right) \approx 0\right] \delta\left[\left(E-\frac{\kappa}{2} A^{+}\right) \approx 0\right] \\
& \times \delta\left[\left(\pi-\partial_{-} \varphi-e A^{+}\right) \approx 0\right] \delta\left[\left(e \partial_{-} \varphi+e^{2} A^{+}\right) \approx 0\right] \delta\left[A^{-} \approx 0\right]
\end{aligned}
$$

The Hamiltonian and path integral quantization of the theory under the gauge: $A^{-} \approx 0$ is now complete.

\section{Light-Front BRST Formulation}

For the BRST formulation of the theory [20-22], we rewrite the theory as a quantum system that possess the generalized gauge invariance called BRST symmetry. For this, we first enlarge the Hilbert space of our gaugeinvariant theory and replace the notion of gauge-transformation, which shifts operators by $c$-number functions, by a BRST transformation, which mixes operators with Bose and Fermi statistics, we then introduce new anti-commuting variables $\mathrm{c}$ and $\bar{c}$ (Grassman numbers on the classical level, operators in the quantized theory) and a commuting variable $b$ such that [20-22]:

$$
\begin{aligned}
& \hat{\delta} \varphi=e c, \hat{\delta} \pi=0, \hat{\delta} A^{+}=-\partial_{-} c, \\
& \hat{\delta} A^{-}=-\partial_{+} c, \hat{\delta} A_{2}=-\partial_{2} c \\
& \hat{\delta} \Pi^{+}=0, \hat{\delta} \Pi^{-}=\frac{\kappa}{2} \partial_{2} c, \hat{\delta} E=-\frac{\kappa}{2} \partial_{-} c, \\
& \hat{\delta} \Pi_{s}=\hat{\delta} \Pi_{u}=\hat{\delta} \Pi_{v}=\hat{\delta} \Pi_{w}=0 \\
& \hat{\delta} s=-\partial_{+} \partial_{+} c, \hat{\delta} u=e \partial_{+} c, \\
& \hat{\delta} v=-\partial_{+} \partial_{2} c, \hat{\delta} w=-\partial_{+} \partial_{-} c \\
& \hat{\delta} c=0, \hat{\delta} \bar{c}=b, \hat{\delta} b=0
\end{aligned}
$$

with the property $\hat{\delta}^{2}=0$. We now define a BRST-in- 
variant function $\mathrm{f}$ (a function of all the phase space variables of the BRST-invariant theory) such that $\hat{\delta} f=0$. Performing gauge-fixing in the BRST formalism implies adding to the first-order Lagrangian density $\mathcal{L}_{I 0}$, a trivial BRST-invariant function. We could thus write e.g.:

$$
\begin{aligned}
\mathcal{L}_{\text {BRST }}= & {\left[\frac{\kappa}{2}\left[-A^{-} \partial_{-} A_{2}+A^{-} \partial_{2} A^{+}-A^{+} \partial_{2} A^{-}+A_{2} \partial_{-} A^{-}\right]-\frac{1}{2}\left(e A_{2}+\partial_{2} \varphi\right)^{2}+\left(\partial_{-} \varphi+e A^{+}\right) \partial_{+} \varphi\right.} \\
& \left.-\frac{\kappa}{2} A_{2} \partial_{+} A^{+}+\frac{\kappa}{2} A^{+} \partial_{+} A_{2}+\hat{\delta}\left[\bar{c}\left(-\partial_{+} A^{-}+\frac{1}{e} \varphi+\frac{1}{2} b\right)\right]\right]
\end{aligned}
$$

The last term in the above equation is the extra BRST-invariant gauge-fixing term. After one integration by parts, the above equation could now be written as:

$$
\begin{aligned}
\mathcal{L}_{\text {BRST }}= & {\left[\frac{\kappa}{2}\left[-A^{-} \partial_{-} A_{2}+A^{-} \partial_{2} A^{+}-A^{+} \partial_{2} A^{-}+A_{2} \partial_{-} A^{-}\right]-\frac{1}{2}\left(e A_{2}+\partial_{2} \varphi\right)^{2}+\left(\partial_{-} \varphi+e A^{+}\right) \partial_{+} \varphi\right.} \\
& \left.-\frac{\kappa}{2} A_{2} \partial_{+} A^{+}+\frac{\kappa}{2} A^{+} \partial_{+} A_{2}+b\left(-\partial_{+} A^{-}+\frac{1}{e} \varphi\right)+\frac{1}{2} b^{2}-\bar{c} \partial_{+} \partial_{+} c-\bar{c} C\right]
\end{aligned}
$$

Proceeeding classically, the Euler Lagrange equation for $b$ reads

$$
b=\left(\partial_{+} A^{-}-\frac{1}{e} \varphi\right)
$$

the requirement $\hat{\delta} b=0$ then implies

$$
\hat{\delta} b=\hat{\delta}\left(\partial_{+} A^{-}\right)-\frac{1}{e} \hat{\delta} \varphi=0
$$

which in turn implies

$$
\partial_{+} \partial_{+} c=-c
$$

The above equation is also an Euler-Lagrange equation (ELE) obtained by the variation of $\mathcal{L}_{B R S T}$ with respect to $\bar{C}$. In introducing momenta one has to be careful in defining those for the fermionic variables. We thus define the bosonic momenta in the usual manner so that

$$
\Pi^{+}:=\frac{\partial}{\partial\left(\partial_{+} A^{-}\right)} \mathcal{L}_{B R S T}=-b
$$

but for the fermionic momenta with directional derivatives we set

$$
\begin{aligned}
& \Pi_{c}:=\mathcal{L}_{\text {BRST }} \frac{\vec{\partial}}{\partial\left(\partial_{+} c\right)}=\partial_{+} \bar{c} ; \\
& \Pi_{\bar{c}}:=\frac{\bar{\partial}}{\partial\left(\partial_{+} \bar{c}\right)} \mathcal{L}_{\text {BRST }}=\partial_{+} c
\end{aligned}
$$

implying that the variable canonically conjugate to $C$ is $\partial_{+} \bar{c}$ and the variable conjugate to $\bar{c}$ is $\partial_{+} c$. For writing the quantum Hamilotonian density from the Lagrangian density in the usual manner we remember that the former has to be Hermitian so that:

$$
\begin{aligned}
\mathcal{H}_{B R S T} & =\left[\pi \partial_{+} \varphi+\Pi^{+} \partial_{+} A^{-}+\Pi^{-} \partial_{+} A^{+}+E_{2} \partial_{+} A_{2}+\Pi_{s} \partial_{+} s+\Pi_{u} \partial_{+} u+\Pi_{v} \partial_{+} v+\Pi_{w} \partial_{+} w+\Pi_{c}\left(\partial_{+} c\right)+\left(\partial_{+} \bar{c}\right) \Pi_{\bar{c}}-\mathcal{L}_{B R S T}\right] \\
& =\left[\Pi_{s} \partial_{+} s+\Pi_{u} \partial_{+} u+\Pi_{v} \partial_{+} v+\Pi_{w} \partial_{+} w-\frac{\kappa}{2}\left[-A^{-} \partial_{-} A_{2}+A^{-} \partial_{2} A^{+}-A^{+} \partial_{2} A^{-}+A_{2} \partial_{-} A^{-}\right]\right. \\
& \left.+\frac{1}{2}\left(e A_{2}+\partial_{2} \varphi\right)^{2}+\Pi^{+}\left(-\partial_{+} A^{-}+\frac{1}{e} \varphi\right)-\frac{1}{2}\left(\Pi^{+}\right)^{2}+\Pi_{c} \Pi_{\bar{c}}+\bar{c} c\right]
\end{aligned}
$$

We can check the consistency of our definitions of the the fermionic momenta by looking at the Hamiltons equations for the Fermionic variables:

$$
\partial_{+} c=\frac{\vec{\partial}}{\partial \Pi_{c}} \mathcal{H}_{B R S T} ; \partial_{+} \bar{c}=\mathcal{H}_{B R S T} \frac{\bar{\partial}}{\partial \Pi_{\bar{c}}}
$$

Thus we see that

$$
\partial_{+} c=\frac{\vec{\partial}}{\partial \Pi_{c}} \mathcal{H}_{B R S T}=\Pi_{\bar{c}}, \partial_{+} \bar{c}=\mathcal{H}_{B R S T} \frac{\bar{\partial}}{\partial \Pi_{\bar{c}}}=\Pi_{c}
$$

is in agreement with our definitions of the fermionic momenta. Also, for the operators $c, \bar{c}, \partial_{+} c$ and $\partial_{+} \bar{c}$, one needs to satisfy the anticommutation relations of $\partial_{+} c$ with $\bar{C}$ or of $\partial_{+} \bar{C}$ with $c$, but not of $c$, with $\bar{C}$. In general, $c$ and $\bar{C}$ are independent canonical variables and one assumes that:

$$
\begin{aligned}
& \left\{\Pi_{c}, \Pi_{\bar{c}}\right\}=\{\bar{c}, c\}=0, \partial_{+}\{\bar{c}, c\}=0, \\
& \left\{\partial_{+} \bar{c}, c\right\}=(-1)\left\{\partial_{+} c, \bar{c}\right\}
\end{aligned}
$$

where $\{$,$\} means an anti-commutator. We thus see that$ 
the anti-commutators in the above equation are nontrivial and need to be fixed. In order to fix these, we demand that c satisfy the Heisenberg equation:

$$
\left[c, \mathcal{H}_{\text {BRST }}\right]=i \partial_{+} c
$$

and using the property $c^{2}=\bar{c}^{2}=0$ one obtains

$$
\left[c, \mathcal{H}_{\text {BRST }}\right]=\left\{\partial_{+} \bar{c}, c\right\} \partial_{+} c
$$

The last three equations then imply:

$$
\left\{\partial_{+} \bar{c}, c\right\}=(-1)\left\{\partial_{+} c, \bar{c}\right\}=i
$$

Here the minus sign in the above equation is nontrivial and implies the existence of states with negative norm in the space of state vectors of the theory. The BRST charge operator $Q$ is the generator of the BRST transformations. It is nilpotent and satisfies $Q^{2}=0$. It mixes operators which satisfy Bose and fermi statistics. According to its conventional definition, its commutators with Bose operators and its anti-commutators with Fermi operators for the present theory satisfy:

$$
\begin{aligned}
& {\left[A^{+}, Q\right]=\left[A^{-}, Q\right]=\left[A_{2}, Q\right]=[\varphi, Q]=\partial_{+} c} \\
& {\left[E_{2}, Q\right]=-\frac{\kappa}{2} \partial_{+}} \\
& {[\pi, Q]=\left(\partial_{-} c+\partial_{-} \partial_{+} c\right),\left[\Pi^{-}, Q\right]=e^{2} c+\left(e-\frac{\kappa}{2}\right) \partial_{+} c(36 \mathrm{~b})} \\
& \{\bar{c}, Q\} \\
& =(-1)\left(\Pi^{+}+\Pi^{-}+E_{2}+\pi-\partial_{-} \varphi-e A^{+}+\frac{\kappa}{2}\left(A_{2}-A^{+}\right)\right)(36 \mathrm{c}) \\
& \left\{\partial_{+} \bar{c}, Q\right\}=(-1)\left(e \partial_{-} \varphi+e^{2} A^{+}\right)
\end{aligned}
$$

All other commutators and anti-commutators involving $Q$ vanish. In view of this, the BRST charge operator of the present theory could be written as:

$$
\begin{aligned}
& Q=\int \mathrm{d}^{2} x\left[i c\left(e \partial_{-} \varphi+e^{2} A^{+}\right)\right. \\
& \left.-i \partial_{+} c\left(\pi+\Pi^{+}+\Pi^{-}+E-\partial_{-} \varphi-e A^{+}+\frac{\kappa}{2}\left(A_{2}-A^{+}\right)\right)\right]
\end{aligned}
$$

This equation implies that the set of states satisfying the conditions:

$$
\begin{aligned}
& \Pi^{+}|\psi\rangle=0,\left(\Pi^{-}+\frac{\kappa}{2} A_{2}\right)|\psi\rangle=0 \\
& \left(E-\frac{\kappa}{2} A^{+}\right)|\psi\rangle=0,\left(\pi-\partial_{-} \varphi-e A^{+}\right)|\psi\rangle=0 \\
& \left(e \partial_{-} \varphi+e^{2} A^{+}\right)|\psi\rangle=0
\end{aligned}
$$

belong to the dynamically stable subspace of states $|\psi\rangle$ satisfying $Q|\psi\rangle=0$, i.e., it belongs to the set of BRST- invariant states. In order to understand the condition needed for recovering the physical states of the theory we write the operators $c$ and $\bar{c}$ in terms of fermionic annihilation and creation operators. For this purpose we consider the equation: $\partial_{+} \partial_{+} c=-c$. The solution of this equation gives the Heisenberg operator $c(\tau)$ where $\tau\left(\equiv x^{+}\right)$is the light-cone time variable, (and correspondingly $\bar{C}(\tau)$ ) as:

$$
c(\tau)=\mathrm{e}^{i \tau} B+\mathrm{e}^{-i \tau} D ; \bar{c}(\tau)=\mathrm{e}^{-i \tau} B^{\dagger}+\mathrm{e}^{i \tau} D^{\dagger}
$$

which at the time $\tau=0$ imply

$$
\begin{aligned}
& c \equiv c(0)=B+D ; \bar{c} \equiv \bar{c}(0)=B^{\dagger}+D^{\dagger} \\
& \partial_{+} c \equiv \partial_{+} c(0)=i(B-D) ; \\
& \partial_{+} \bar{c} \equiv \partial_{+} \bar{c}(0)=-i\left(B^{\dagger}-D^{\dagger}\right)
\end{aligned}
$$

By imposing the conditions

$$
\begin{aligned}
& c^{2}=\bar{c}^{2}=\{\bar{c}, c\}=\left\{\partial_{+} \bar{c}, \partial_{+} c\right\}=0 \\
& \left\{\partial_{+} \bar{c}, c\right\}=i=-\left\{\partial_{+} c, \bar{c}\right\}
\end{aligned}
$$

one then obtains

$$
\begin{aligned}
& B^{2}+\{B, D\}+D^{2}=B^{\dagger^{2}}+\left\{B^{\dagger}, D^{\dagger}\right\}+D^{\dagger^{2}}=0 \\
& \left\{B, B^{\dagger}\right\}+\left\{D, D^{\dagger}\right\}+\left\{B, D^{\dagger}\right\}+\left\{B^{\dagger}, D\right\}=0 \\
& \left\{B, B^{\dagger}\right\}+\left\{D, D^{\dagger}\right\}-\left\{B, D^{\dagger}\right\}-\left\{B^{\dagger}, D\right\}=0 \\
& \left\{B, B^{\dagger}\right\}-\left\{D, D^{\dagger}\right\}-\left\{B, D^{\dagger}\right\}+\left\{D, B^{\dagger}\right\}=-1 \\
& \left\{B, B^{\dagger}\right\}-\left\{D, D^{\dagger}\right\}+\left\{B, D^{\dagger}\right\}-\left\{D, B^{\dagger}\right\}=-1
\end{aligned}
$$

with the solution

$$
\begin{aligned}
& B^{2}=D^{2}=B^{\dagger^{2}}=D^{\dagger^{2}}=0 \\
& \{B, D\}=\left\{B^{\dagger}, D\right\}=\left\{B, D^{\dagger}\right\}=\left\{B^{\dagger}, D^{\dagger}\right\}=0 \\
& \left\{B^{\dagger}, B\right\}=-\frac{1}{2} ;\left\{D^{\dagger}, D\right\}=\frac{1}{2}
\end{aligned}
$$

We now let $|0\rangle$ denote the fermionic vacuum for which

$$
B|0\rangle=D|0\rangle=0
$$

Now by defining $|0\rangle$ to have norm one, we have

$$
\left\langle 0\left|B B^{\dagger}\right| 0\right\rangle=\frac{-1}{2},\left\langle 0\left|D D^{\dagger}\right| 0\right\rangle=\frac{1}{2}
$$

so that

$$
\left.\left.B^{\dagger} \mid\right)\right\rangle \neq 0, D^{\dagger}|0\rangle \neq 0
$$

The theory is thus seen to possess negative norm states in the fermionic sector. The existence of these negative norm states as free states of the fermionic part of $\mathcal{H}_{\text {BRST }}$ 
is however, irrelevant to the existence of physical states in the orthogonal subspace of the Hilbert space. In terms of fermionic annihilation and creation operators the quantum Hamiltonian density is:

$$
\begin{aligned}
\mathcal{H}_{\text {BRST }}= & {\left[\Pi_{s} \partial_{+} s+\Pi_{u} \partial_{+} u+\Pi_{v} \partial_{+} v+\Pi_{w} \partial_{+} w+2\left(B^{\dagger} B+D^{\dagger} D\right)\right.} \\
& \left.-\frac{\kappa}{2}\left[-A^{-} \partial_{-} A_{2}+A^{-} \partial_{2} A^{+}-A^{+} \partial_{2} A^{-}+A_{2} \partial_{-} A^{-}\right]+\frac{1}{2}\left(e A_{2}+\partial_{2} \varphi\right)^{2}+\Pi^{+}\left(-\partial_{+} A^{-}+\frac{1}{e} \varphi\right)-\frac{1}{2}\left(\Pi^{+}\right)^{2}\right]
\end{aligned}
$$

and the BRST charge operator is:

$$
\begin{aligned}
Q= & \int \mathrm{d}^{2} x\left[i B\left[e \partial_{-} \varphi+e^{2} A^{+}-i\left(\pi+\Pi^{+}+\Pi^{-}+E-\partial_{-} \varphi-e A^{+}+\frac{\kappa}{2}\left(A_{2}-A^{+}\right)\right)\right]\right. \\
& \left.+i D\left[e \partial_{-} \varphi+e^{2} A^{+}+i\left(\pi+\Pi^{+}+\Pi^{-}+E-\partial_{-} \varphi-e A^{+}+\frac{\kappa}{2}\left(A_{2}-A^{+}\right)\right)\right]\right]
\end{aligned}
$$

Now because $Q|\psi\rangle=0$, the set of states annihiliated by $Q$ contains not only the set for which the constraints of the theory hold but also additional states for which

$$
\begin{aligned}
& B|\psi\rangle=D|\psi\rangle=0 \\
& \Pi^{+}|\psi\rangle \neq 0,\left(\Pi^{-}+\frac{\kappa}{2} A_{2}\right)|\psi\rangle \neq 0 \\
& \left(E-\frac{\kappa}{2} A^{+}\right)|\psi\rangle \neq 0,\left(\pi-\partial_{-} \varphi-e A^{+}\right)|\psi\rangle \neq 0 \\
& \left(e \partial_{-} \varphi+e^{2} A^{+}\right)|\psi\rangle \neq 0
\end{aligned}
$$

The Hamiltonian is also invariant under the anti-BRST transformation given by:

$$
\begin{aligned}
& \overline{\hat{\delta}} \varphi=-e \bar{c}, \overline{\hat{\delta}} \pi=0, \overline{\hat{\delta}} A^{+}=\partial_{-} \bar{c} \\
& \overline{\hat{\delta}} A^{-}=\partial_{+} \bar{c}, \overline{\hat{\delta}} A_{2}=\partial_{2} \bar{c} \\
& \overline{\hat{\delta}} \Pi^{+}=0, \overline{\hat{\delta}} \Pi^{-}=-\frac{\kappa}{2} \partial_{2} \bar{c}, \overline{\hat{\delta}} E=\frac{\kappa}{2} \partial_{-} \bar{c} \\
& \overline{\hat{\delta}} \Pi_{s}=\overline{\hat{\delta}} \Pi_{u}=\overline{\hat{\delta}} \Pi_{v}=\overline{\hat{\delta}} \Pi_{w}=0 \\
& \overline{\hat{\delta}} s=\partial_{+} \partial_{-} \bar{c}, \overline{\hat{\delta}} u=-e \partial_{+} \bar{c} \\
& \overline{\hat{\delta}} v=\partial_{+} \partial_{2} \bar{c}, \overline{\hat{\delta}} w=\partial_{+} \partial_{-} \bar{c} \\
& \overline{\hat{\delta}} \bar{c}=0, \overline{\hat{\delta}}_{c}=-b, \overline{\hat{\delta}} b=0
\end{aligned}
$$

with generator or anti-BRST charge

$$
\bar{Q}=\int \mathrm{d}^{2} x\left[-i \bar{c}\left(e \partial_{-} \varphi+e^{2} A^{+}\right)+i \partial_{+} \bar{c}\left(\pi+\Pi^{+}+\Pi^{-}+E-\partial_{-}-e A^{+}+\frac{\kappa}{2}\left(A_{2}-A^{+}\right)\right)\right]
$$

which in terms of annihilation and creation operators reads:

$$
\begin{aligned}
Q= & \int \mathrm{d}^{2} x\left[-i B^{?}\left[e \partial_{-} \varphi+e A^{+}-i\left(\pi+\Pi^{+}+\Pi^{-}+E-\partial_{-} \varphi-e A^{+}+\frac{\kappa}{2}\left(A_{2}-A^{+}\right)\right)\right]\right. \\
& \left.-i D^{\dagger}\left[e \partial_{-} \varphi+e^{2} A^{+}+i\left(\pi+\Pi^{+}+\Pi^{-}+E-\partial_{-} \varphi-e A^{+}+\frac{\kappa}{2}\left(A_{2}-A^{+}\right)\right)\right]\right]
\end{aligned}
$$

We also have

$$
\partial_{+} Q=\left[Q, \mathcal{H}_{\text {BRST }}\right]=0 ; \partial_{+} \bar{Q}=\left[\bar{Q}, \mathcal{H}_{\text {BRST }}\right]=0
$$

with

$$
\mathcal{H}_{\text {BRST }}=\int \mathrm{d}^{2} \chi \mathcal{H}_{\text {BRST }}
$$

and we further impose the dual condition that both $Q$ and $\bar{Q}$ annihilate physical states, implying that:

$$
Q|\psi\rangle=0 \text { and } ; \bar{Q}|\psi\rangle=0
$$

The states for which the constraints of the theory hold, satisfy both of these conditions and are in fact, the only states satisfying both of these conditions, since although with (43),

$$
2\left(B^{\dagger} B+D^{\dagger} D\right)=-2\left(B B^{\dagger}+D D^{\dagger}\right)
$$

there are no states of this operator with $B^{\dagger}|\psi\rangle=0$ and $D^{\dagger}|\psi\rangle=0$, and hence no free eigenstates of the fermionic part of $\mathcal{H}_{B R S T}$ that are annihiliated by each of 
$B, B^{\dagger}, D$, and $D^{\dagger}$. Thus the only states satisfying $\bar{Q}|\psi\rangle=0$ and $\bar{Q}|\psi\rangle=0$ are those that satisfy the constraints of the theory. Now because $Q|\psi\rangle=0$, the set of states annihilated by $Q$ contains not only the set of states for which the constraints of the theory hold but also additional states for which the constraints of the theory do not hold in particular. This situation is, however, easily avoided by aditionally imposing on the theory, the dual condition: $\bar{Q}|\psi\rangle=0$ and $\bar{Q}|\psi\rangle=0$. Thus by imposing both of these conditions on the theory simultaneously, one finds that the states for which the constraints of the theory hold satisfy both of these conditions and, in fact, these are the only states satisfying both of these conditions because in view of the conditions on the fermionic variables $c$ and $\bar{c}$ one cannot have simultaneously $c, \partial_{+} c$ and $\bar{c}, \partial_{+} \bar{c}$, applied to $|\psi\rangle$ to give zero. Thus the only states satisfying $Q|\psi\rangle=0$ and $\bar{Q}|\psi\rangle=0$ are those that satisfy the constraints of the theory and they belong to the set of BRST-invariant as well as to the set of anti-BRST-invariant states.

Alternatively, one can understand the above point in terms of fermionic annihiliation and creation operators as follows. The condition $Q|\psi\rangle=0$ implies that the set of states annihiliated by $Q$ contains not only the states for which the constraints of the theory hold but also additional states for which the constraints do not hold. However, $\bar{Q}|\psi\rangle=0$ guarantees that the set of states annihiliated by $\bar{Q}$ contains only the states for which the constraints hold, simply because $B^{\dagger}|\psi\rangle \neq 0$ and $D^{\dagger}|\psi\rangle \neq 0$. Thus in this alternative way also we see that the states satisfying $Q|\psi\rangle=\bar{Q}|\psi\rangle=0$ are only those states that satisfy the constraints of the theory and also that these states belong to the set of BRST-invariant and anti-BRST-invariant states. This completes the BRST formulation of the theory.

\section{Summary and Discussion}

In this work we have presented the complete details of the LF Hamiltonian, path integral and BRST formulations of the CSH theory on the hyperplanes defined by the light-cone (LC) time:

$$
\left(\tau \equiv x^{+}=\frac{\left(x^{0}+x^{1}\right)}{\sqrt{2}}=\text { constant }\right)
$$

$[23,24]$, in the BSP of the Higgs potential. The preliminary results of our present investigations are given in Ref. [13].

Further, our present studies are in contrast with our earlier work of Ref. [12] on the quantization of the same theory, where we have studied the IFQ of this theory on the hyperplanes defined by the IF time:

$$
\left(x^{0}=t=\text { constant }\right)
$$

in the BSP of the Higgs potential (instead of its LFQ). In the present work, on the other hand, we have studied its LFQ on the hyperplanes defined by the LC time:

$$
\left(\tau \equiv x^{+}=\frac{\left(x^{0}+x^{1}\right)}{\sqrt{2}}=\text { constant }\right) \text {. }
$$

In the BSP of the Higgs potential, the phase $\varphi\left(x^{\mu}\right)$ of the complex matter field $\Phi\left(x^{\mu}\right)$ carries the charge degree of freedom of the complex matter field and is, in fact, akin to the Goldstone boson [12-15]. The theory in the so-called symmetry phase of the Higgs potential has also been studied by us earlier [9-11]. Also, different aspects of this theory have been studied by several authors in various contexts [1-8]. For a comparative description of different studies, we refer to the work of Refs. [1-8], as well as to our earlier work of Refs. [7-13].

What actually necessitates our present studies is an important fact that the LFQ of any theory has several advantages over its IFQ for several well known reasons $[23,24]$. Also because the LF coordinates are not related to the conventional IF coordinates by a finite Lorentz transformation, the descriptions of the same physical result may be different in the IF and LF dynamics. In fact, the study of a theory using the IFQ as well as the LFQ determines the canonical structure and the constrained dynamics of the theory rather completely $[18,19]$.

The LFQ has several advantages over the conventional IFQ $[23,24]$. In particular, for a LF theory seven out of ten Poincare generators are kinematical while the IF theory has only six kinematical generators. In our treatment, we have made the convention to regard the light-cone variable $x^{+} \equiv \tau$ as the LF time coordinate $[23,24]$ and the light-cone variable $x^{-}$has been treated as the longitudinal spatial coordinate $[23,24]$. The temporal evolution of the system in $x^{+}$is generated by the total Hamiltonian of the system. If we consider the invariant distance between two spacetime points in $(2+1)$-dimension $[23,24]$ :

$$
\begin{aligned}
& (x-y)^{2}:=\left[\left(x^{0}-y^{0}\right)^{2}-\left(x^{1}-y^{1}\right)^{2}-\left(x^{2}-y^{2}\right)^{2}\right] \\
& (\text { IFQ }) \\
& (x-y)^{2}:=\left[2\left(x^{+}-y^{+}\right)\left(x^{-}-y^{-}\right)-\left(x^{2}-y^{2}\right)^{2}\right] \\
& (\text { LFQ })
\end{aligned}
$$

then we find that in the instant-form, the points on the $x^{0}=y^{0}=$ constant hyperplanes, have space-like separation except when they are coincident when it becomes light-like one. On the light-front, however, with

$x^{+}=y^{+}=$constant, the distance becomes independent of $\left(x^{-}-y^{-}\right)$and the separation again becomes spacelike. The LF field theory therefore does not necessarily 
need to be local in $x^{-}$, even if the corresponding instant-form theory is formulated as a local one. The nonvanishing equal-time commutators of the IF theory are nonlocal and nonvanishing for space-like distances and violate the microcausality principle $[23,24]$. The nonvanishing equal light-cone-time commutators for the present theory, on the otherhand would be nonlocal in the light-cone space variable $x^{-}$and nonvanishing only on the light-cone. There would therefore be no conflict with the microcausality principle for the LF theory unlike the case of the equal-time commutators in the IF theory. For further details on the Dirac's different relativistic forms of dynamics, we refer to the work of Refs. [23,24].

The constrained dynamics of the present theory in IFQ as studied by us in Ref. [12], reveals that the theory possesses a set of four constraints where three constraints are primary and one secondary Gauss law constraint. The matrix of the Poission brackets of these two constraints is singular and therefore they form a set of first-class constraints, implying in turn, that the corresponding theory is gauge-invariant. The theory is indeed seen to possess a local vector gauge symmetry. For further details of this work, we refer to the work of Ref. [12].

The LFQ of this theory, on the other hand, reveals that the LF theory possesses a set of five constraints where four constraints are primary and one is a secondary Gauss law constraint. The matrix of the Poission brackets of these five constraints is singular implying that they form a set of first-class constraints. This implies in turn, that the corresponding theory is gauge-invariant. The theory is indeed seen to possess a local vector gauge symmetry, and correspondingly there exists a conserved local vector gauge current.

Now because the set of constraints of the theory is first-class, one could quantize the theory under some suitable gauge-fixing as we have done in our present work for the Hamiltonian and path integral quantization of our theory. For this we have choosen the gauge-fixing: $A^{-} \approx 0$. The gauge $A^{-} \approx 0$ here represents the lightcone coulomb gauge. This gauge choice is not only acceptable and consistent with our quantization procedures but is also physically more intersting gauge choice representing the light-cone coloumb gauge.

However, in the above Hamiltonian and path integral quantization of the theory under some gauge-fixing conditions the gauge-invariance of the theory gets broken because the procedure of gauge-fixing converts the set of first-class constraints of the theory into a set of second-class one, by changing the matrix of the Poission brackets of the constraints of the theory from a singular one into a non-singular one. In view of this, in order to achieve the quantization of our gauge-invariant theory, such that the gauge-invariance of the theory is maintained even under gauge-fixing, one of the possible ways is to go to a more generalized procedure called the BRST quantization [20-22], where the extended gauge symmetry called as the BRST symmetry is maintained even under gauge-fixing. It is therefore desirable to achieve this so-called BRST quantization also if possible. This therefore makes a kind of complete quantization of a theory. The light-cone BRST quantization of the present theory has been studied by us in the present work, under some specfic gauge choice (where a particular gauge has been choosen by us and which is not unique by any means). In this procedure, when we embed the original gauge-invariant theory into a BRST system, the quantum Hamiltonian density $\mathcal{H}_{B R S T}$ (which includes the gauge-fixing contribution) commutes with the BRST charge as well as with the anti-BRST charge. The new (extended) gauge symmetry which replaces the gauge invariance is maintained (even under the BRST gauge-fixing) and hence projecting any state onto the sector of BRST and anti-BRST invariant states yields a theory which is isomorphic to the original gauge-invariant theory.

\section{Acknowledgements}

This work was supported in part by the National Science Foundation under Grant No. PHY0904782 and the Department of Energy under Grant No. DE-FG0287ER40371.

\section{REFERENCES}

[1] G. V. Dunne, “Aspects of Chern-Simons Theories," Lectures Given at Les Houches Summer School in Theoretical Physics, Session 69: Topological Aspects of LowDimensional Systems, Les Houches, 7-31 July 1998. arXiv: hep-th/9902115

[2] F. Wilczek, "Quantum Mechanics of Fractional Spin Particles," Physical Review Letters, Vol. 49, No. 14, 1982, pp. 957-959. doi:10.1103/PhysRevLett.49.957

[3] D. Boyanovsky, E. T. Newman and C. Rovelli, "On the Quantization of Dynamical Systems with Chern-Simons Terms," Physical Review D, Vol. 45, No. 4, 199, pp. 1210-1216. doi:10.1103/PhysRevD.45.1210

[4] E. J. Ferrer, R. Hurka and V. de la Incera, "High Temperature Anyon Superconductivity," Modern Physics Letters B, Vol. 11, No. 1, 1997, pp. 1-8. doi:10.1142/S0217984997000025

[5] R. B. Laughlin, "Nobel Lecture: Fractional Quantization," Reviews Modern Physics, Vol. 71, No. 4, 1999, pp. 863874. doi:10.1103/RevModPhys.71.863

[6] A. Smirnov, "Notes on Csern-Simons Theory in the Temporal Gauge," International School of Subnuclear Physics (ISSP 2009): 47th Course: The Most Unexpected at LHC and the Status of High Energy Frontier, 29 August-7 September, 2009, Sicily, pp. 489-498. arXiv:0910.5011[hep-th]

[7] U. Kulshreshtha and D. S. Kulshreshtha, "Hamiltonian, 
Path Integral and BRST Formulations of the ChernSimons Theory under Appropriate Gauge-Fixing," Canadian Journal of Physics, Vol. 86, No. 2, 2008, pp. 401407. doi:10.1139/P07-176

[8] U. Kulshreshtha, D. S. Kulshreshtha and J. P. Vary, "Light-Front Hamiltonian, Path Integral and BRST Formulations of the Chern-Simons Theory under Appropriate Gauge-Fixing," Journal of Modern Physics, Vol. 1, 2010, pp. 385-392. doi:10.4236/jmp.2010.16055

[9] U. Kulshreshtha, D. S. Kulshreshtha, H. J. W. MuellerKirsten and J. P. Vary, "Hamiltonian, Path Integral and BRST Formulations of the Chern-Simons Higgs Theory under Appropriate Gauge-Fixing," Physica Scripta, Vol. 79, No. 4, 2009, Article ID: 045001. doi:10.1088/0031-8949/79/04/045001

[10] U. Kulshreshtha, "Light-Front Quantization of the ChernSimons Higgs Theory," Invited Contributed Talk at the International Light-Cone Conference (LC2010) on Relativistic Hadronic and Particle Physics, Valencia, 18-22 May 2010.

[11] U. Kulshreshtha, D. S. Kulshreshtha and J. P. Vary, "Light-Front Hamiltonian, Path Integral and BRST Formulations of the Chern-Simons Higgs Theory under Appropriate Gauge-Fixing," Physica Scripta, Vol. 82, No. 5, 2010, Article ID: 055101. doi:10.1088/0031-8949/82/05/055101

[12] U. Kulshreshtha, "Hamiltonian, Path Integral and BRST Formulations of the Chern-Simons Higgs Theory in the Broken Symmetry Phase," Physica Scripta, Vol. 75, No. 6, 2007, pp. 795-802. doi:10.1088/0031-8949/75/6/009

[13] U. Kulshreshtha, "Light-Front Quantization of the ChernSimons-Higgs Theory in the Broken Symmetry Phase," Invited Contributed Talk at the International Light-Cone Conference: LC2011, Dallas, 22-27 June 2011, pp. 457461.

[14] U. Kulshreshtha and D. S. Kulshreshtha, "The FrontForm Hamiltonian and BRST Formulations of the Nielsen-Olesen Model in The Broken Symmetry Phase," Ca- nadian Journal of Physics, Vol. 82, 2004, pp. 569-583.

[15] D. Boyanovsky, "Chern-Simons with Matter Fields: Broken Symmetry Phase," Physical Review D, Vol. 42, No. 4, 1990, pp. 1179-1183. doi:10.1103/PhysRevD.42.1179

[16] P. A. M. Dirac, "Generalized Hamiltonian Dynamics," Canadian Journal of Mathematics, Vol. 2, 1950, pp. 129. 148. doi:10.4153/CJM-1950-012-1

[17] P. Senjanovic, "Path Integral Quantization of Field Theories with Second Class Constraints," Annals of Physics, Vol. 100, No. 1-2, 1976, pp. 227-261.

[18] U. kulshreshtha and D. S. Kulshreshtha, "Conformally Gauge-Fixed Polyakov D1 Brane Action in the Presence of a 2-Form Gauge Field: The Instant-Form and FrontForm Hamiltonian and Path Integral Formulations," Physical Letters B, Vol. 555, No. 3-4, 2003, pp. 255-263.

[19] U. Kulshreshtha, D. S. Kulshreshtha and J. P. Vary, "Light-Front Hamiltonian and Path Integral Formulations of Large N Scalar $\mathrm{QCD}_{2}$," Physics Letters B, Vol. 708, No. 1-2, 2012, pp. 195-198. doi:10.1016/j.physletb.2012.01.034

[20] C. Becchi, A. Rouet and A. Stora, "The Abelian HiggsKibble Model. Unitarity of the S-Operator," Physics Letters B, Vol. 52, No. 3, 1974, pp. 344-346. doi:10.1016/0370-2693(74)90058-6

[21] V. Tyutin, "Lebedev Report No. FIAN-39," (unpublished).

[22] D. Nemeschansky, C. Preitschopf and M. Weinstein, "A BRST Primer," Annals of Physics, Vol. 183, No. 2, 1988, pp. 226-268. doi:10.1016/0003-4916(88)90233-3

[23] P. A. M. Dirac, "Forms of Relativistic Dynamics," Reviews Modern Physics, Vol. 21, No. 3, 1949, pp. 392-399. doi:10.1103/RevModPhys.21.392

[24] S. J. Brodsky, H. C. Pauli and S. S. Pinsky, "Quantum Chromodynamics and Other Field Theories on the LightCone," Physics Reports, Vol. 301, No. 4-6, 1998, pp. 299-486. doi:10.1016/S0370-1573(97)00089-6 